\title{
Comparative simulation studies of multipacting in higher-order-mode couplers of superconducting rf cavities
}

\author{
Y. M. Li, ${ }^{1,2}$ K. X. Liu, ${ }^{1, *}$ and R. L. Geng ${ }^{2, \dagger}$ \\ ${ }^{1}$ State Key Laboratory of Nuclear Physics and Technology, Institute of Heavy Ion Physics, \\ Peking University, Beijing, 100871, China \\ ${ }^{2}$ Thomas Jefferson National Accelerator Facility, Newport News, Virginia 23606, USA
}

(Received 3 July 2013; published 20 February 2014)

\begin{abstract}
Multipacting (MP) in higher-order-mode (HOM) couplers of the International Linear Collider (ILC) baseline cavity and the Continuous Electron Beam Accelerator Facility (CEBAF) $12 \mathrm{GeV}$ upgrade cavity is studied by using the ACE3P suites, developed by the Advanced Computations Department at SLAC. For the ILC cavity HOM coupler, the simulation results show that resonant trajectories exist in three zones, corresponding to an accelerating gradient range of $0.6-1.6 \mathrm{MV} / \mathrm{m}, 21-34 \mathrm{MV} / \mathrm{m}, 32-35 \mathrm{MV} / \mathrm{m}$ and $>40 \mathrm{MV} / \mathrm{m}$, respectively. For the CEBAF $12 \mathrm{GeV}$ upgrade cavity HOM coupler, resonant trajectories exist in one zone, corresponding to an accelerating gradient range of 6-13 MV $/ \mathrm{m}$. Potential implications of these MP barriers are discussed in the context of future high-energy pulsed as well as medium-energy continuous wave accelerators based on superconducting radio frequency cavities. Frequency scaling of MP's predicted in HOM couplers of the ILC, CEBAF upgrade, Spallation Neutron Source (SNS), and Free-Electron Laser in Hamburg (FLASH) third harmonic cavity is given and found to be in good agreement with the analytical result based on the parallel plate model.
\end{abstract}

DOI: 10.1103/PhysRevSTAB.17.022002

PACS numbers: 52.80.Pi, 52.20.Dq, 79.20.Hx

\section{INTRODUCTION}

Multipacting (MP) is a phenomenon of resonant electron multiplication in which some specific inner surface areas of a radio frequency (rf) device are repeatedly bombarded by electrons that gain energy from the rf field contained in the device [1,2]. MP was observed in superconducting radio frequency (SRF) cavities for accelerators in the 1970s and has attracted many studies since then $[3,4]$. Because of the energy deposition of MP electrons at bombardment sites, the cavity wall temperature will rise. In case the SRF transition temperature is exceeded, this would lead to a thermal runaway and ultimately rapid dissipation of the stored energy in the cavity. MP was used to constitute a limitation to the reachable accelerating gradient in a SRF cavity. Progress has been made over the past decades in understanding and alleviating the MP problem in SRF cavities. For example, by adopting an elliptical profile in an axially symmetric cavity operated in the TM010 mode, "hard" MP's [5] in which electrons may gain energy of hundreds of electronvolts, can be fully avoided [6,7]. This has allowed successful applications of elliptical SRF cavities for electron acceleration (in linacs such as CEBAF and FLASH and

\footnotetext{
*kxliu@pku.edu.cn

'geng@jlab.org
}

Published by the American Physical Society under the terms of the Creative Commons Attribution 3.0 License. Further distribution of this work must maintain attribution to the author $(s)$ and the published article's title, journal citation, and DOI. storage rings such as the Transposable Ring Intersecting Storage Accelerator in Nippon (TRISTAN), the Hadron Electron Ring Anlage (HERA), the Cornell Electron Storage Ring (CESR), B-factory of High Energy Accelerator Research Organization (KEKB), the LHC, etc.) as well as for proton acceleration (in linacs such as SNS).

Higher order modes (HOM) excitation in a rf cavity by charged particles has some adverse effects such as causing emittance growth of the beam under acceleration or resulting in additional heat load to the cryogenics in case of a SRF accelerator. Hence HOM damping and extraction are needed to avoid beam instability and to reduce unwanted heat load to the cryogenic system of a SRF accelerator. Various HOM dampers have been developed and found successful applications. A commonly used HOM damper in a SRF cavity is a coaxial-type coupler, such as the one originally developed for the $1300 \mathrm{MHz}$ Tera Electron Volt Energy Superconducting Linear Accelerator (TESLA) cavity [8]. A sketch is shown in Fig. 1. The TESLA-style HOM coupler has been adopted also in SRF cavities for the SNS, the energy doubling of CEBAF from 6 to $12 \mathrm{GeV}$, the European X-Ray Free Electron Laser (XFEL), and the proposed International Linear Collider (ILC).

Through systematic numerical simulation studies, the present work deals with the MP characteristics of HOM couplers of the ILC baseline cavity and the CEBAF upgrade cavity. Our studies are motivated by some recently observed experimental phenomena both at Jefferson Lab and elsewhere in TESLA-style 9-cell cavities. Those phenomena are not yet understood but suspected to be 


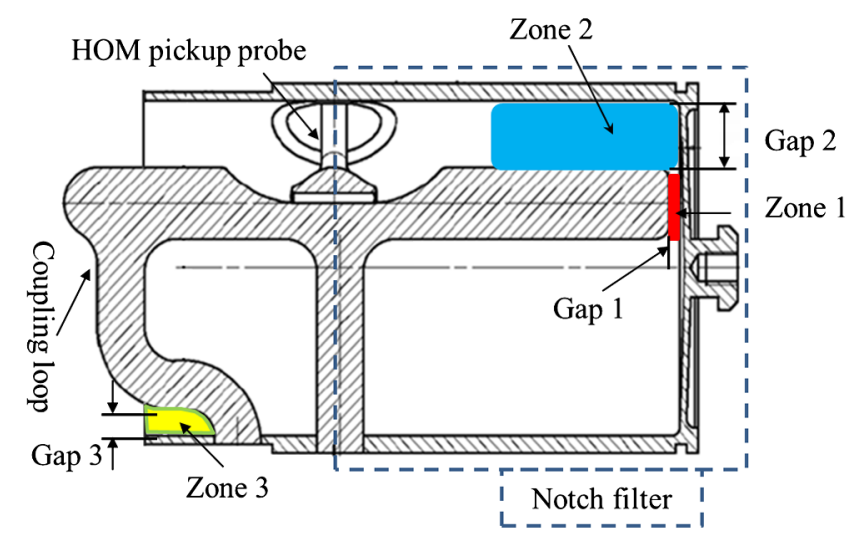

FIG. 1. TESLA-style coaxial HOM coupler. Three major parts including the coupling loop, the notch filter, and the HOM pickup probe are shown. Also shown are definition of three gaps and three zones (with color) for characterization of MP.

related to MP's in HOM couplers. Examples include the baking induced field emission turn-on phenomenon at a gradient of $>23 \mathrm{MV} / \mathrm{m}$ [9] and the pass-band mode excitation phenomenon with the detection of only lowenergy $\mathrm{x}$-rays [10]. In addition, the ILC baseline design requires an average gradient of $31.5 \mathrm{MV} / \mathrm{m}$ for cavities in operation with a beam with an allowable gradient spread of $\pm 20 \%$. This means that an ILC cavity may need to run at a gradient of up to $38 \mathrm{MV} / \mathrm{m}$. Because of the gradient overhead consideration, a cavity operated with a beam at $38 \mathrm{MV} / \mathrm{m}$ must be tested up to $42 \mathrm{MV} / \mathrm{m}$ during its vertical qualification testing [11]. Previous simulations of MP in HOM couplers of a TESLA-type cavity were limited to a gradient of $35 \mathrm{MV} / \mathrm{m}$ [12]. Therefore the present study explores a broader gradient range of up to $47.5 \mathrm{MV} / \mathrm{m}$. Lastly, our studies also concern MP in HOM couplers of a CEBAF upgrade cavity. Eighty of these cavities are to be ultimately installed in CEBAF to double its energy from $6 \mathrm{GeV}$ to $12 \mathrm{GeV}$ [13]. A detailed analysis of MP characteristics of the HOM couplers in a CEBAF upgrade cavity is still lacking. It should be noted that there are differences in the physical configurations between the HOM couplers for an ILC cavity and CEBAF upgrade cavity [14]. Therefore it is of great interest to compare the MP characteristics of the two.

\section{COAXIAL-TYPE HOM COUPLER}

As shown in Fig. 1, the main parts of a TESLA-style coaxial-type HOM coupler include (1) a loop antenna for coupling the HOM power; (2) a notch filter for rejection of the power due to the accelerating mode; (3) a HOM pickup probe for extracting HOM's to an external load. The notch filter is tuned to be at resonance with the fundamental mode of the cavity so as to suppress fundamental mode coupling to the HOM pickup probe. For an ILC cavity, two HOM couplers are located with one on each side of the cavity end group. In the case of a CEBAF $12 \mathrm{GeV}$ upgrade cavity, two HOM couplers are located on one side of the cavity end group $[15,16]$. The HOM couplers of SNS cavities are also based on the TESLA-type HOM coupler [17].

\section{SIMULATION TOOL}

The electromagnetic code suites ACE3P developed at SLAC are based on a high-order parallel finite element method for geometry fidelity and simulation accuracy. ACE3P includes the eigenmode solver Omega3P and the particle tracking code Track3P [18]. In our simulation studies, Omega3P was used to obtain the field distribution in the cavity and HOM couplers and Track3P was used for calculation of trajectories of MP electrons. These codes are previously used in studying MP in SRF cavities with favorable results $[19,20]$.

Here a brief introduction of the MP algorithm is given [21]. First, electrons are launched from specific surfaces at different $\mathrm{rf}$ phases. The initial velocity of the electron is perpendicular to the surface. The initial energy is fixed (typically $2 \mathrm{eV}$ ). The initially launched electrons follow trajectories determined by the electromagnetic fields and eventually hit the boundary of the structure. Secondary electrons are then emitted, the number of which depends on the secondary emission yield (SEY) of the boundary material and the impact energy. Tracking of electrons continues until a resonant trajectory is identified. The present study concerns predominantly the one-surface and two-surface MP. In these cases, a resonant trajectory is found when the following two criteria are met: (1) electrons return to a small area repeatedly at a nearly constant phase angle (numerically some spread in phase angle on the order of a few degrees is allowed); (2) the SEY corresponding to the impact energy is greater than unity. Typically the tracking time is long enough to ensure a predetermined number of impacts are registered. The kinetic energy of electrons upon each impact is also

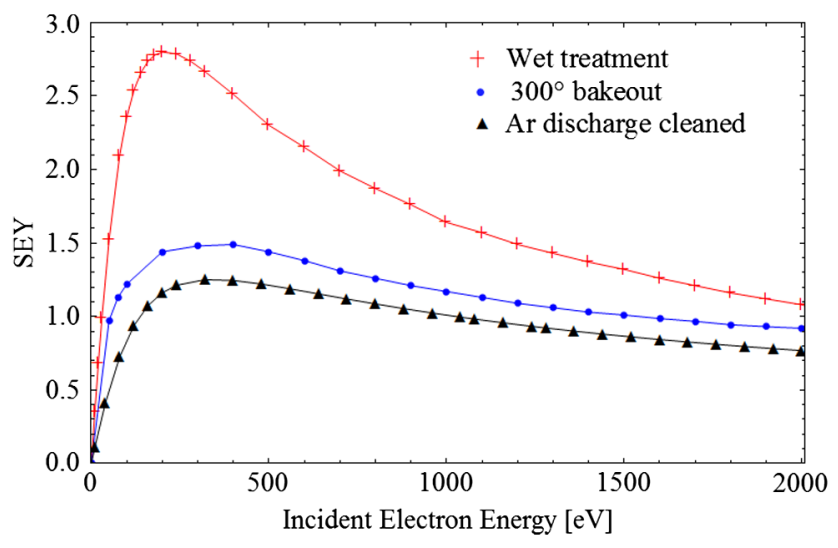

FIG. 2. Secondary emission yield curves for niobium surfaces of various treatments. 
registered for each involved surface. For resonant electrons hitting the same surface, their impact energy is average and plotted against the acceleration gradient. In our simulation, the niobium SEY curve is shown in Fig. 2 [22]. The SEY includes the contribution from true secondary electrons as well as reflected electrons and rediffused electrons. The effect of SEY enhancement due to glance incidence is presently not considered in the code. A very useful concept of enhanced counter function (ECF) is used for analyzing the MP data. The ECF is a measure of total number of electrons after several rf periods.

\section{SIMULATION RESULTS}

\section{A. ILC cavity HOM couplers}

For the purpose of MP simulations in HOM couplers, we adopt a simplified model as shown in Fig. 3. It consists of a one-cell TESLA cavity (end half-cell shape) and a HOM coupler. The validity of this model is justified in viewing that the electromagnetic field configuration in the HOM coupler region is uniquely proportional to the field in the end cell of a 9-cell cavity. The subtle difference between the center halfcell and the end half-cells is expected to introduce negligible effect for the MP characteristics in HOM couplers.

The notch filter rf behavior is very sensitive to the notch gap. Before the MP simulation, the notch frequency has to be tuned to the cavity resonance frequency. Details about tuning the notch gap can be found in Ref. [23]. The notch filter gap in our model is tuned to $1.41 \mathrm{~mm}$.

The rf electric field distribution of the fundamental mode in the HOM can is shown in Fig. 4. The ratio of the local peak electric field $E_{\mathrm{pt}}$ at the tip of the HOM antenna to the accelerating gradient $E_{\mathrm{pt}} / E_{\mathrm{acc}}$ is $\sim 27 \%$. At an accelerating gradient of $42 \mathrm{MV} / \mathrm{m}$, the peak electric field in the HOM coupler region amounts to $>11 \mathrm{MV} / \mathrm{m}$, which is clearly not a trivial field in terms of MP or even field emission consideration.

The initial electrons are launched from the whole inner surface of the HOM coupler. After a comprehensive survey, we found MP's in three zones as indicated in Fig. 1. Detailed information is given in the following paragraphs.

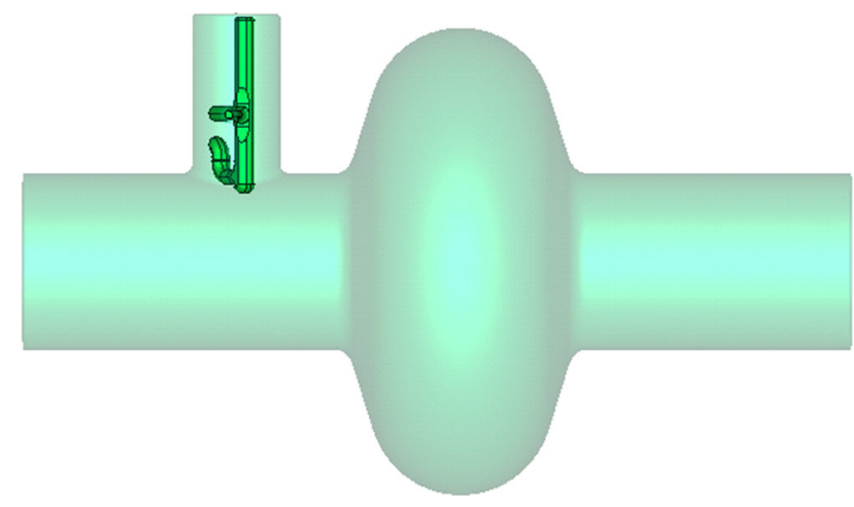

FIG. 3. One-cell cavity model for MP simulations in an ILC cavity.

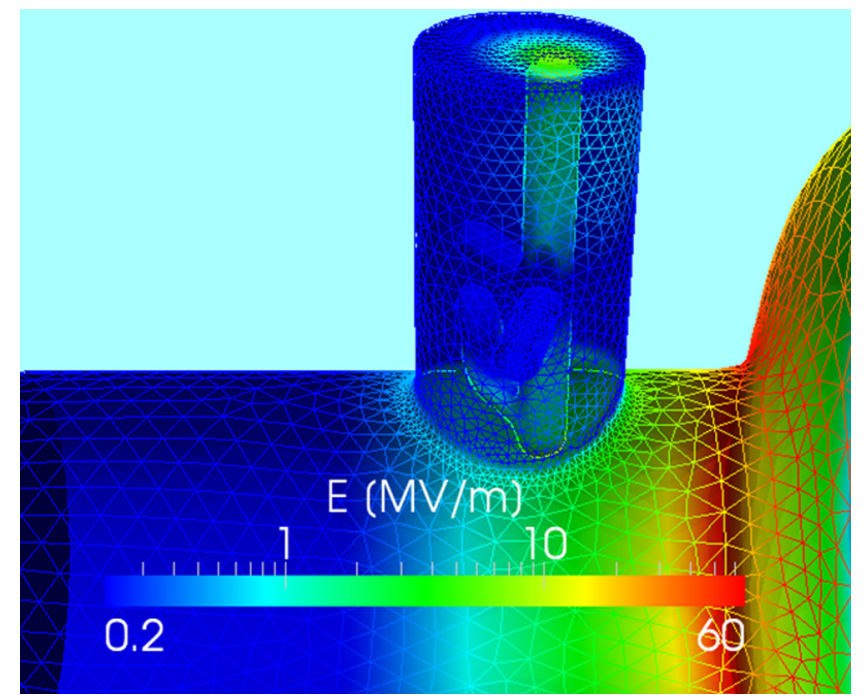

FIG. 4. Contour of the electric field in a TESLA-style HOM coupler $\left(E_{\mathrm{acc}}=31.5 \mathrm{MV} / \mathrm{m}\right)$. Rapid decay of the electric field of the fundamental mode from the end cell (at right) into the beam tube (at left) is evident. The peak local electric field at the tip of the coupling loop is $27 \%$ of the accelerating gradient. The peak electric field across the gap (gap 1 in Fig. 1) between the antenna and the bottom of the HOM can is $19 \%$ of the accelerating gradient.

The ratio of the peak electric field $E_{\mathrm{pg}}$ across the gap in zone 1 (between the HOM antenna and the HOM can, see Fig. 1) to the accelerating gradient $E_{\mathrm{pg}} / E_{\text {acc }}$ is $\sim 19 \%$. The rf magnetic field vanishes in zone 1. As a result, the MP in zone 1 can be treated as the well-known configuration of a pair of parallel plates, with an average gap of $1.41 \mathrm{~mm}$ and slope angle of $2^{\circ}$. Our numerical simulation results show that the first-order MP takes place at an accelerating gradient in the range of $0.6-1.6 \mathrm{MV} / \mathrm{m}$. The impact energy of the MP electrons is in the range of $50-325 \mathrm{eV}$ as shown in Fig. 5(a). The ECF (for sustained impacting of $20 \mathrm{rf}$ periods) for different SEY's are given in Fig. 5(b). Clearly, even for the case of a "clean" niobium surface, there is a good chance of MP in zone 1.

Typical electron trajectories of MP electrons are given in Fig. 5(c). It can be seen that the MP in zone 1 is not a fixed two-point MP between two parallel plates. Because of the fringe field effect that resulted from the asymmetric structure of the HOM coupler, MP electrons drift away from the gap space and ultimately fall off the square area of the inner conductor.

In zone 2, the gap between the inner conductor and the HOM can is $8.2 \mathrm{~mm}$ (gap 2 in Fig. 1) by design. Resonant trajectories exist in zone 2 for an accelerating gradient range of 21-35 MV/m. The electron impact energy for the sustained impacting of 10, 20, and 30 times is given in Fig. 6(a). As can be seen, sustained impacting for 10 times is possible over a broad gradient range of $21-35 \mathrm{MV} / \mathrm{m}$ with an impacting energy in the range of $40-2500 \mathrm{eV}$. However, sustained impacting for 20 times is only possible 

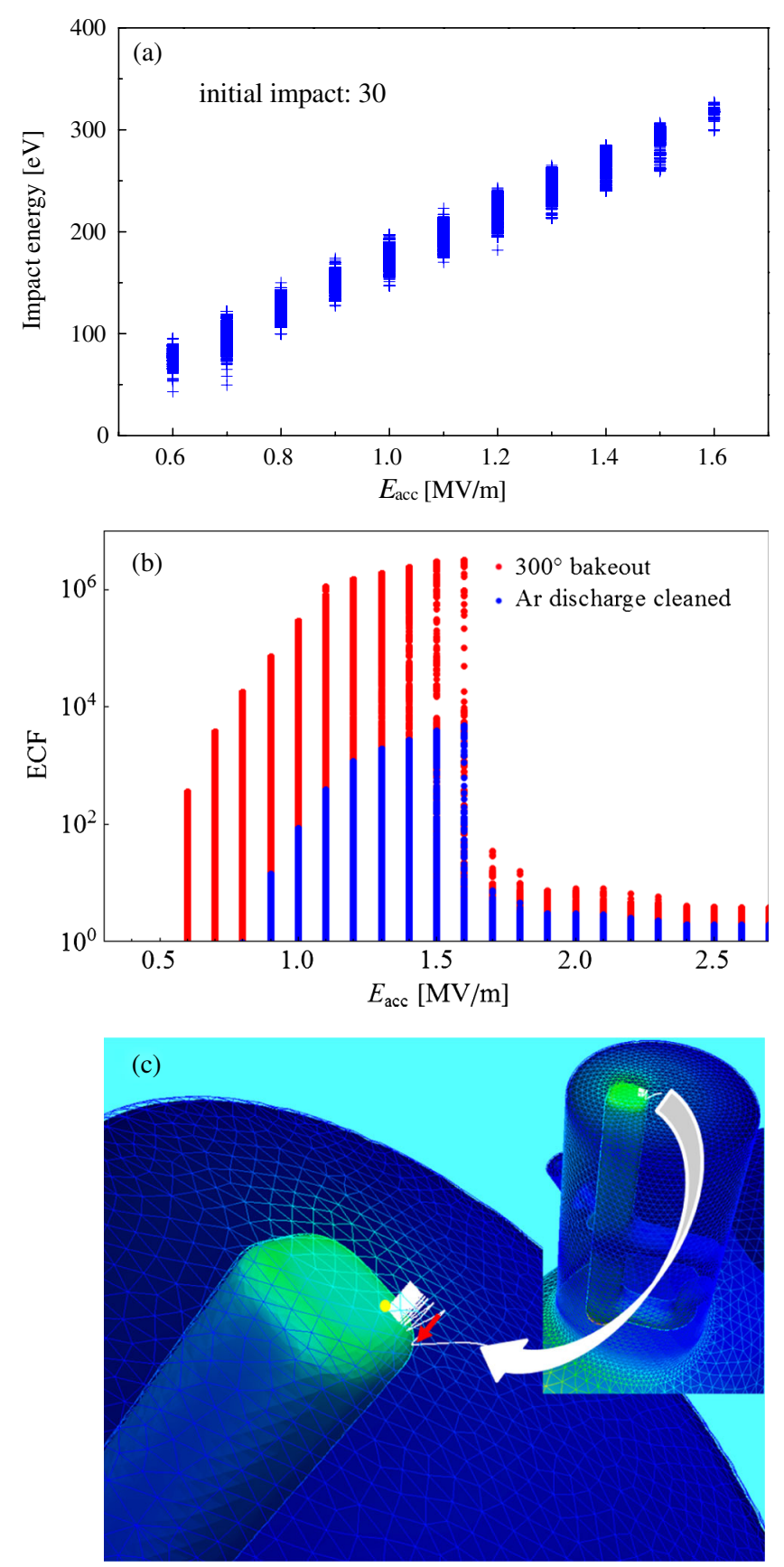

FIG. 5. Characteristics of zone $1 \mathrm{MP}$ : (a) electron impact energy; (b) enhancement counter function after impacting for 20 rf periods; (c) typical electron trajectory. The emission site is indicated by the yellow dot. The movement direction of the MP electron is indicated by red arrows. Finally, the emission electron slips out of the square area of the inner conductor.

for a reduced gradient range of $21-25 \mathrm{MV} / \mathrm{m}$, around $28 \mathrm{MV} / \mathrm{m}$ and 32-34 MV/m with an impacting energy in the range of 40-1100 eV. The gradient range for which sustained impacting for 30 times is further narrowed to 32-34 MV/m with an impacting energy in the range of 400-800 eV. The enhanced counter function (for $20 \mathrm{rf}$
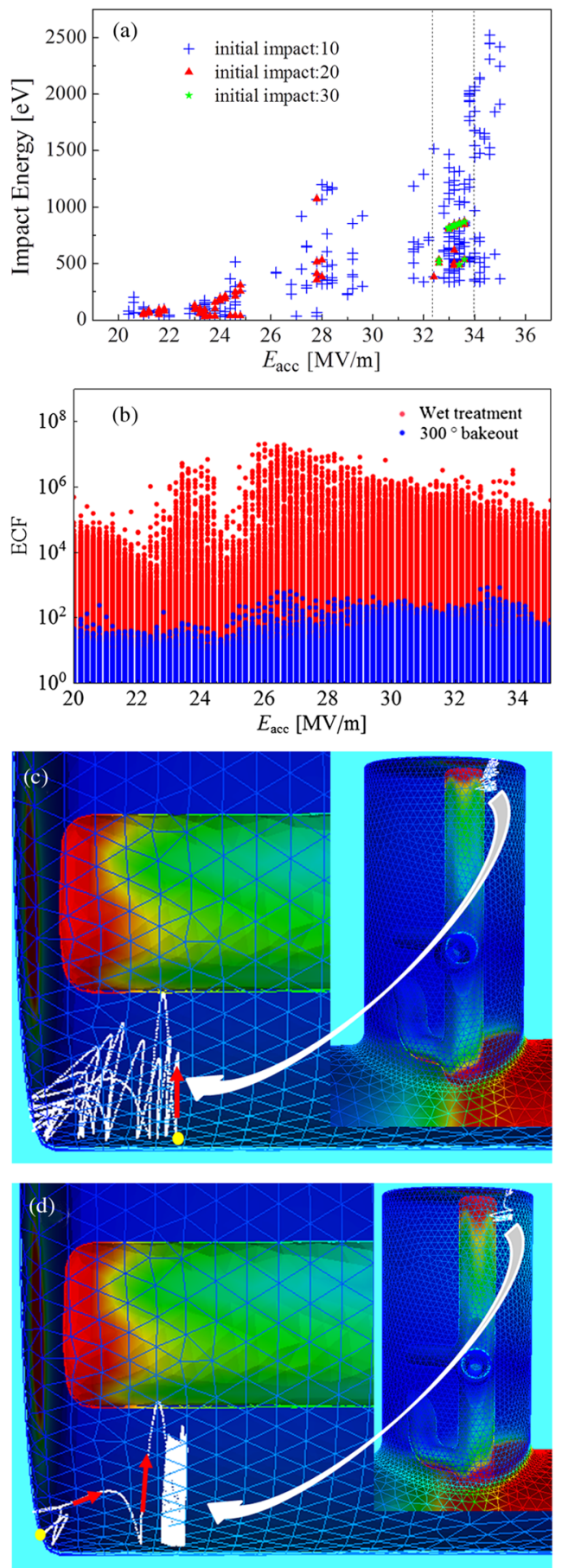

FIG. 6. Characteristics of zone 2 MP: (a) electron impact energy; (b) ECF after impacting for $20 \mathrm{rf}$ periods; (c) typical electron trajectories of quasiresonant MP for gradients in the range of $21-30 \mathrm{MV} / \mathrm{m}$. The initial emission electron is from the yellow dot position moving forward with the red arrow direction. The emission electrons will ultimately slip out of the resonant phase after some number of impacts and ultimately get lost. (d) Typical electron trajectories of "hard" MP for gradients in the range of $32-35 \mathrm{MV} / \mathrm{m}$. The initial emission electron is from the yellow dot position moving forward with the direction shown in the figure. Finally, the emission electrons form a stale trajectory. 

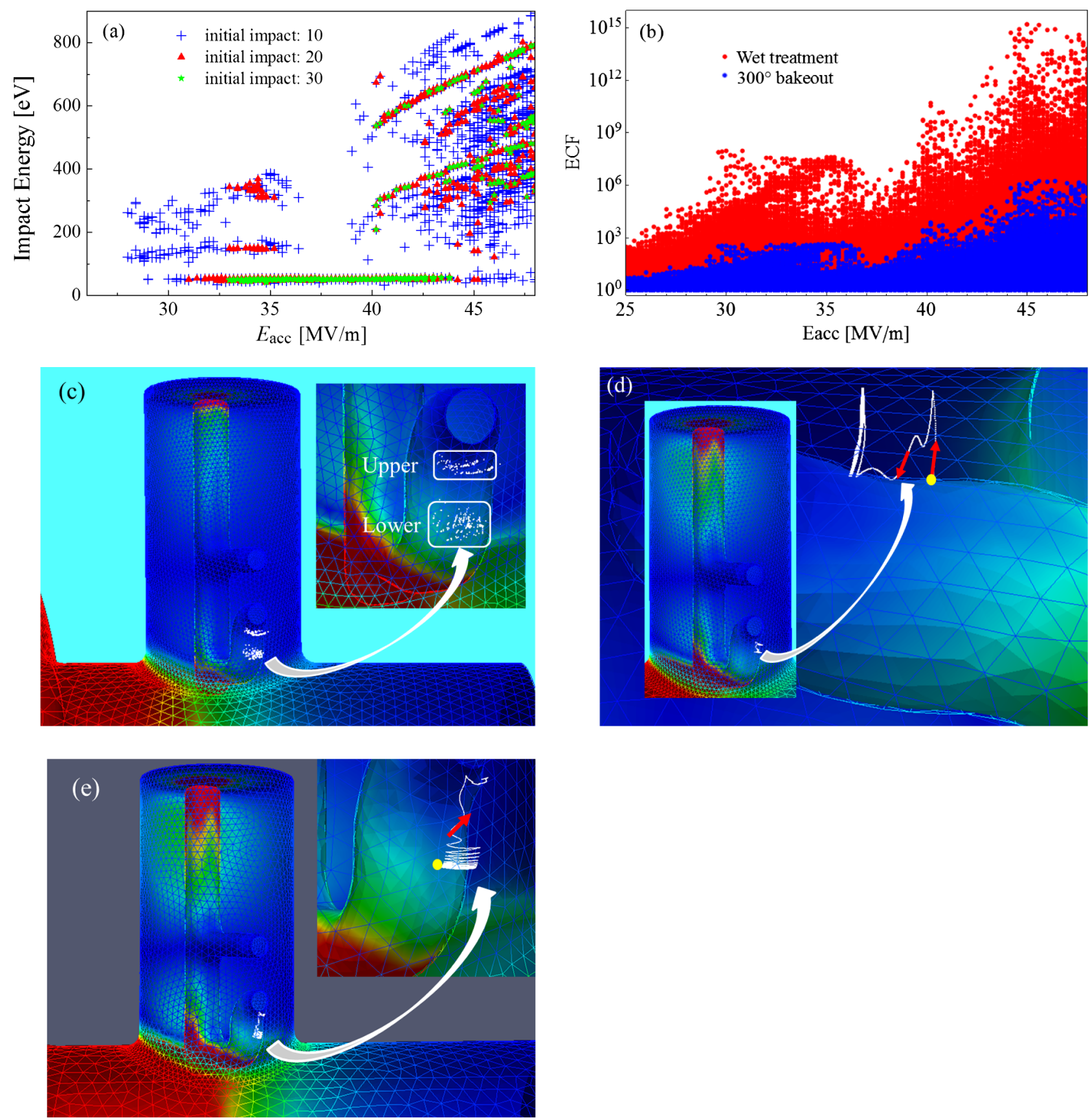

FIG. 7. Characteristics of zone 3 MP: (a) electron impact energy; (b) enhancement counter function after 20 rf periods; (c) two MP regions; (d) typical low impact energy resonant electron (upper region) trajectories. The emission site is indicated by the yellow dot. The initial movement direction of the MP electron is indicated by red arrows. The ultimate resonant trajectory between the two surfaces with impact energy of $\sim 50 \mathrm{eV}$ is also shown. (e) Typical high impact energy resonant electron (lower region) trajectories. The MP electron ultimately slips out of resonance.

periods) for different SEY's is given in Fig. 6(b). Detailed analysis of simulation results shows that zone 2 MP can be divided into two groups. The first MP is from 21-30 MV/m with typical electron trajectories as shown in Fig. 6(c). Because of its complex nature of the trajectories, we term this type of MP as "corner MP," in which more than two points are involved and perhaps it involves some level of hybrid between a one-point MP and a two- point MP. As a result, the MP electron will ultimately slip out of the resonant phase after some number of impacts and ultimately get lost. This kind of "quasiresonant" barrier typically is not expected to be observable for a clean surface with a low SEY because the growth in electron density is limited by multiplication time. Even if the surface cleanliness is less ideal, such a barrier is expected to be only observable at the initial power rise during rf testing. The 
second MP is from $32-34 \mathrm{MV} / \mathrm{m}$ and the typical electron trajectories are shown in Fig. 6(d). This is a first-order one-point MP with resonant trajectories that are sustained even after 30 impacts. In addition, as shown in Fig. 6(a), the impact energy for this MP is in the range of $400-800 \mathrm{eV}$. As a result, this kind of "hard" barrier can persist even after extended "conditioning" by deliberately parking the rf field at the barrier gradients.

In zone 3 , resonant trajectories exist for an accelerating gradient in the range of $32-35 \mathrm{MV} / \mathrm{m}$ and $>40 \mathrm{MV} / \mathrm{m}$. The electron impact energy for sustained impacting of 10 , 20, and 30 times is given in Fig. 7(a). The gradient range for sustained MP becomes narrowed as the impact number increases. The ECF after 20 rf periods is shown in Fig. 7(b). Through detailed analysis, the resonant electrons are found to impact surfaces in two regions shown in Fig. 7(c). The electron impact energy in the upper region tends to be less than $100 \mathrm{eV}$. This barrier is a two-surface third-order MP that involves the inner surface of the HOM can and the outer surface of the HOM loop antenna. Typical upper region electron (around $50 \mathrm{eV}$ ) trajectories (which can persist even after $30 \mathrm{rf}$ cycles) are shown in Fig. 7(d). The electron impact energy in the lower region falls in the range
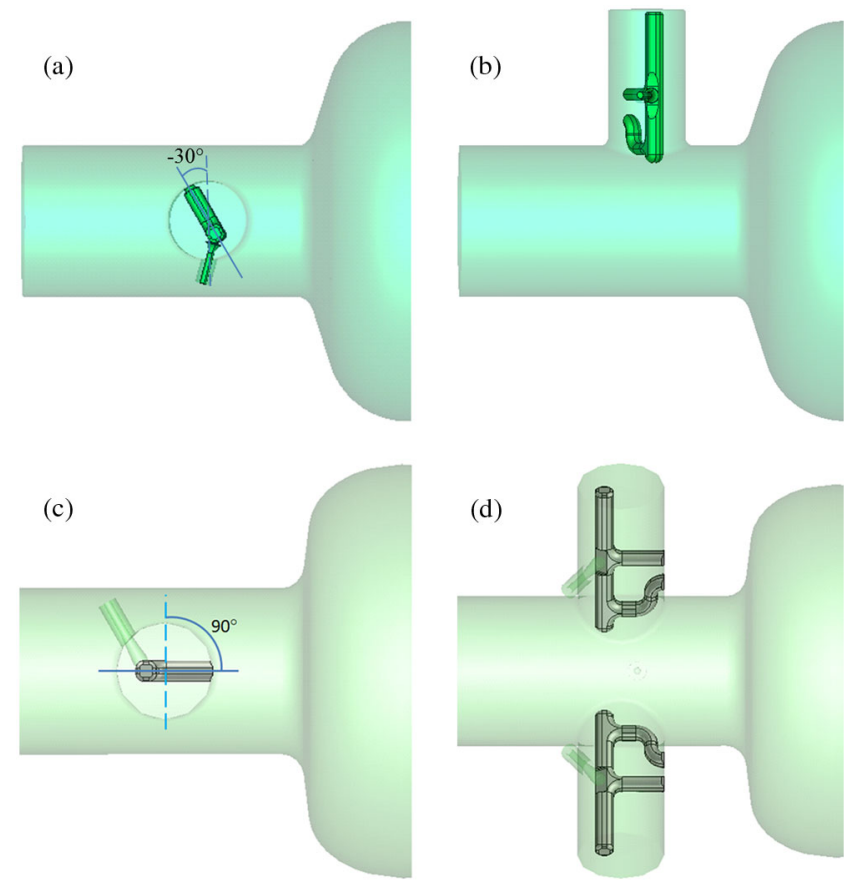

FIG. 8. Comparison of HOM couplers in ILC cavity (a),(b) and in CEBAF 12 Gev upgrade cavity (c),(d). The angle between HOM coupler plane and the plane vertical to the cavity axis is $-30^{\circ}$ in ILC cavity and $90^{\circ}$ in CEBAF cavity. The ILC cavity has two HOM couplers, one each at two end-groups with an angular displacement of $115^{\circ}$ with respect to each other when viewed along the cavity axis direction. The CEBAF upgrade cavity has two HOM couplers, both at the same end-group with an angular displacement of $115^{\circ}$ when viewed along the cavity axis direction. of $250-800 \mathrm{eV}$. This barrier is a two-surface third-order MP for gradients below $40 \mathrm{MV} / \mathrm{m}$ and a two-surface first-order MP with gradient above $40 \mathrm{MV} / \mathrm{m}$. Typical high-energy MP electron trajectories (in the lower region) are shown in Fig. 7(e), which shows electrons drafting out of the resonant phase eventually after some number of impacts. Because of the high impacting energy, the hard MP barrier at $>40 \mathrm{MV} / \mathrm{m}$ is likely to persist even after sustained $\mathrm{rf}$ conditioning because of a high SEY (see Fig. 2).

\section{B. CEBAF $12 \mathrm{GeV}$ upgrade cavity HOM coupler}

The HOM couplers in a CEBAF $12 \mathrm{GeV}$ upgrade cavity, as shown in Fig. 8, are based on the TESLA-type HOM coupler design. In comparison to the ILC cavity, the arrangement of the HOM couplers is different because (1) two HOM couplers are placed on one side of the cavity and (2) the HOM antenna tip is farther away from the end-cell cavity. As a result, the coupling of the fundamental mode between the HOM couplers and the end cell is reduced. This reduces the HOM heating and is understood to be an important change for the CW operation of these cavities [14]. The ratio of the local peak electric field at the tip of the HOM antenna to the accelerating gradient $E_{\mathrm{pt}} / E_{\mathrm{acc}}$ is $\sim 8 \%$ (27\% for the ILC cavity). The ratio of the peak electric field across the gap
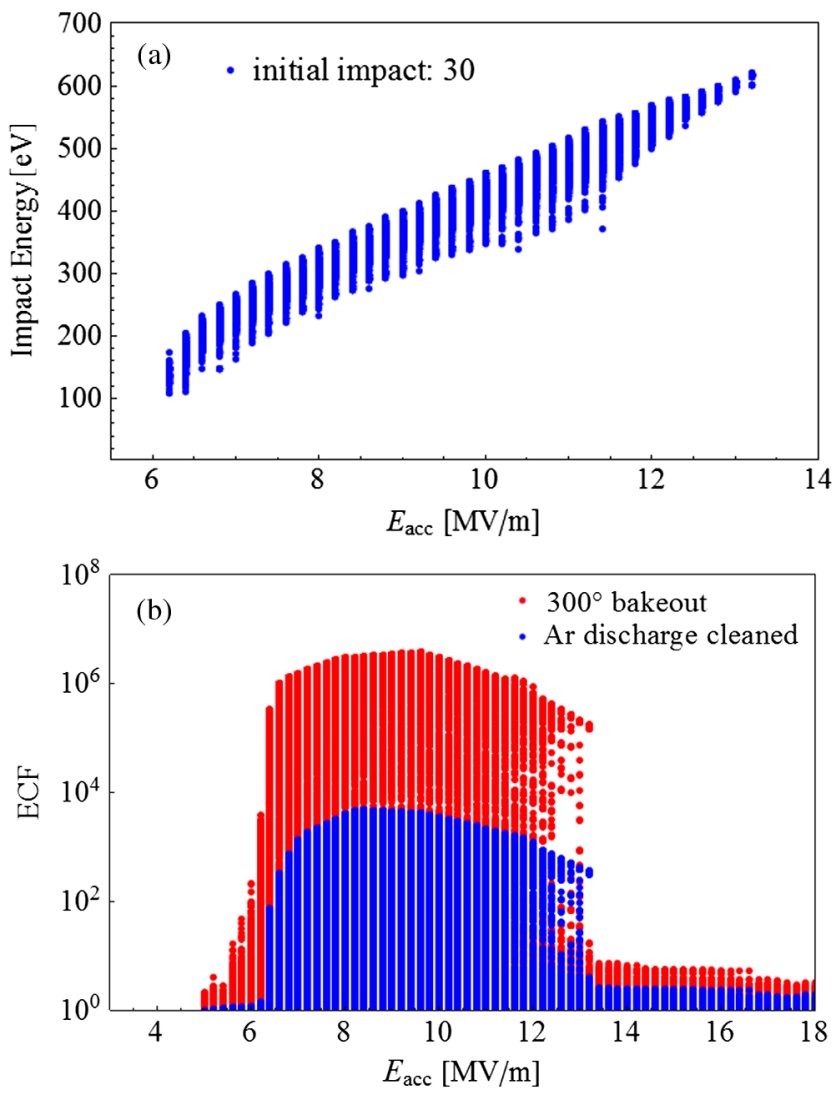

FIG. 9. Characteristics of zone $1 \mathrm{MP}$ in the HOM coupler of a CEBAF upgrade cavity: (a) electron impact energy; (b) ECF after 20 rf periods. 
in zone 1 to the accelerating gradient $E_{\mathrm{pg}} / E_{\mathrm{acc}}$ is $\sim 4.1 \%$ (19\% for the ILC cavity).

Simulation results show that resonant trajectories exist in zone 1 for an accelerating gradient in the range of 6-13 MV/m. In this region, the gap between the HOM antenna and HOM can is tuned to $1.79 \mathrm{~mm}$. The electron impact energy is shown in Fig. 9. This simulation result agrees well with the analytical prediction for a two-surface MP across a pair of parallel plates [24]. This MP barrier corresponds to the one for the gradient range of $0.6-1.6 \mathrm{MV} / \mathrm{m}$ in an ILC cavity.

No MP is identified in zone 2 or zone 3 up to a gradient of $30 \mathrm{MV} / \mathrm{m}$ studied in this work (the operating gradient for CEBAF upgrade cavities is $19.2 \mathrm{MV} / \mathrm{m}$ ).

\section{DISCUSSIONS}

\section{A. Overview and comparison of MP in HOM coupler of ILC and CEBAF upgrade cavity}

The MP characteristics of the TESLA cavity HOM couplers were numerically studied previously by Gonin et al. [12]. Those works predicted two barriers, one at $E_{\text {acc }} 1.1-1.7 \mathrm{MV} / \mathrm{m}$ in zone 1 and the other at $E_{\text {acc }} \sim$ $33.8 \mathrm{MV} / \mathrm{m}$ in zone 2 . These results were compared with the available experimental data. It was stated that a good agreement was observed between the simulation results and the experimental data. More recently, new evidence about zone $1 \mathrm{MP}$ was revealed by Kostin et al. during the XFEL prototype module tests. Their experimental result demonstrated the existence of a MP barrier at $E_{\text {acc }} 1.1 \mathrm{MV} / \mathrm{m}$. Numerical simulation studies were also carried out, predicting a MP barrier in zone 1 at a gradient in the range of $1.5-1.8 \mathrm{MV} / \mathrm{m} \mathrm{[25].}$

A summary of those prior results and our new simulation results is given in Fig. 10. In addition, the analytical result base on the parallel-plate model is also shown. From Ref. [24], the two-surface first-order MP occurs in the gradient range between the lower bound $E_{\min }$,

$$
E_{\min }=\frac{m \omega^{2} d}{\sqrt{4+\pi^{2}} e},
$$

and the upper bound $E_{\max }$,

$$
E_{\max }=\frac{m \omega^{2} d}{2 e},
$$

where $e$ is the electron charge and $m$ the electron mass, and $d$ is the gap between surfaces.

It should be mentioned that there is a slight difference in the zone 1 gap length as used by different workers. In our model, the nominal notch filter gap $1.73 \mathrm{~mm}$ is tuned to $1.41 \mathrm{~mm}$ for reasons given in Sec. IV A. No tuning attempt was reported in Refs. $[12,25]$ where the nominal gap length $1.73 \mathrm{~mm}$ was used instead. This results in a higher $E_{\text {acc }}$ (more than $\sim 50 \%$ ) for the same zone $1 \mathrm{MP}$ barrier by Gonin and Kostin as compared to the present work.

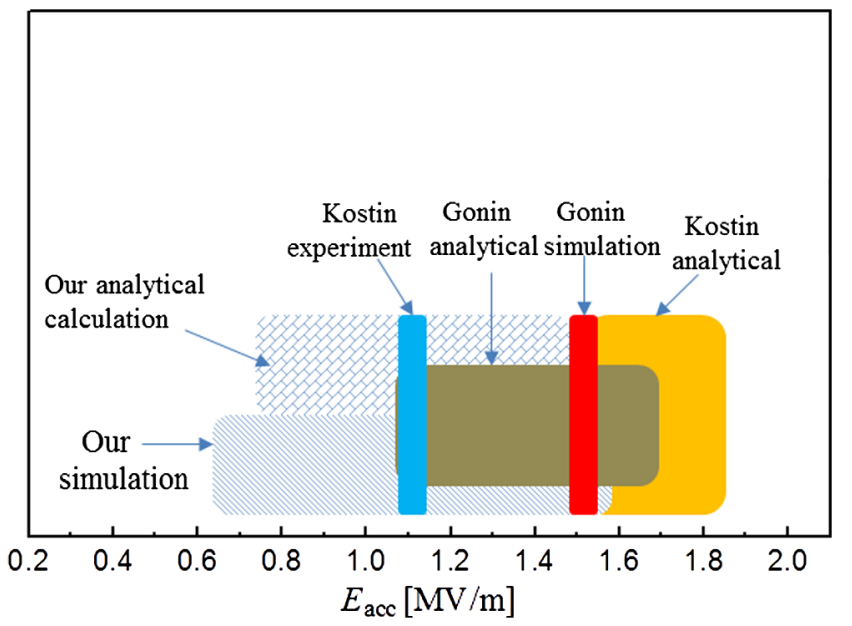

FIG. 10. Summary of zone $1 \mathrm{MP}$ in the TESLA-style cavity HOM coupler. The Kostin experiment data did not give the MP field range in Ref. [25], so we use a narrow bar to represent the data point. The gradient width of Gonin simulation result was also not reported. A narrow bar was used to represent the data point.

Aside from the zone $1 \mathrm{MP}$ at $E_{\text {acc }} 0.6-1.6 \mathrm{MV} / \mathrm{m}$, our simulations also predicted MP's over a broad gradient range from 21 to more than $45 \mathrm{MV} / \mathrm{m}$ in zone 2 and zone 3 in ILC cavities. It must be mentioned that most of the predicted MP's in zone 2 and zone 3 are "quasiresonant" barriers, namely, the resonant impact is able to last for only certain periods of time. Practically, these barriers are not expected to be observable unless the effective SEY of the surface is very high. Nevertheless, even a fleeting MP could cause a burst of gas desorption from the surface in the HOM coupler. In turn, re-condensation of gases on the high electric field region near iris could trigger field emission. This might be one explanation to the experimentally observed phenomenon of baking induced field emission turn-on at a gradient of $>23 \mathrm{MV} / \mathrm{m}$ in electropolished ILC cavities [9]. Further studies of baking effect on the SEY of electropolished niobium surfaces are needed to confirm this scenario.

More importantly, our simulations predict two "hard" MP barriers, one in zone 2 at a gradient in the range of $32-34 \mathrm{MV} / \mathrm{m}$ and the other in zone 3 at $>40 \mathrm{MV} / \mathrm{m}$. Previously, Gonin et al. predicted a MP barrier at $E_{\text {acc }} \sim$ $33.8 \mathrm{MV} / \mathrm{m}$ in zone 2 , which is in agreement with our prediction at 32-34 MV/m. We predict a new barrier at $>40 \mathrm{MV} / \mathrm{m}$. Reports on experimental observation of these predicted MP's are still scarce, suggesting these narrow barriers may be sensitive to the detailed dimension of the actual cavity and the HOM coupler. Nevertheless, as the ILC baseline design average gradient is $31.5 \mathrm{MV} / \mathrm{m}$, which is rather close to the predicted zone $2 \mathrm{MP}$, further experimental studies of this MP barrier are needed due to its significance to the beam operation of ILC cavities. For ILC $1 \mathrm{TeV}$ upgrade, higher gradients are needed. This pushes the cavity toward the $50 \mathrm{MV} / \mathrm{m}$ regime. Our work suggests that MP in the HOM couplers is a potential limit to the 
achievable gradient in a full-scale TESLA-style cavity. Practically, some full-scale 9-cell cavities have reached a gradient of $>40 \mathrm{MV} / \mathrm{m}$. The record gradient is $45 \mathrm{MV} / \mathrm{m}$ [26]. It is therefore possible to experimentally study the predicted MP barrier in zone 3 of the HOM coupler in a TESLA-style cavity.

Our simulation also predicts a MP barrier in the gradient range of $6-13 \mathrm{MV} / \mathrm{m}$ in zone 1 of a CEBAF upgrade cavity. Until now, all 80 cavities for CEBAF $12 \mathrm{GeV}$ upgrade have completed vertical testing and no hard barrier was observed during cryogenic vertical tests. However, some high radiation starting at a gradient as low as $10 \mathrm{MV} / \mathrm{m}$ was observed in some cavities during horizontal tests in cryomodules. Further investigation with regard to the possible role of the zone $1 \mathrm{MP}$ in these cavity behaviors would be interesting.

\section{B. MP in HOM coupler of cavities at other frequencies}

MP in HOM couplers of cavities at other frequencies has been previously observed and studied. In this paper, further simulation studies of two examples have been carried out. This includes the SNS high-beta cavity and the original FLASH third harmonic cavity. A frequency scaling attempt is herein made to analyze the MP behaviors in HOM couplers of cavities at a variety of frequencies.

During the commissioning and operation of the SNS superconducting linac, the high-beta $805 \mathrm{MHz}$ cavities exhibited MP from 9.5 to $18.6 \mathrm{MV} / \mathrm{m}$ and abnormal signals originating from the HOM coupler were observed. Simulations done by Gonin and co-worker using ANALYST showed MP from 10 to $26 \mathrm{MV} / \mathrm{m}$ in zone 3 [12]. Our simulation results predict two barriers in zone 3 at gradient $11-19 \mathrm{MV} / \mathrm{m}$ (third-order MP) and $25-35 \mathrm{MV} / \mathrm{m}$ (first-order MP), respectively. There is a reasonably good agreement between the first barrier we predict and previous experiment and simulation results. The second barrier we predict is above the experimentally achieved gradient, preventing direct comparison.

MP barriers in zone 1 and zone 3 were observed also in HOM couplers of the original $3.9 \mathrm{GHz} 9$-cell cavity. During the high power test of the No. 2 cavity, thermal breakdown was observed in the HOM coupler with the first barrier occurring at about $1-2 \mathrm{MV} / \mathrm{m}$ and the second wider and stronger barrier from 12 to $19 \mathrm{MV} / \mathrm{m}$ [27]. Simulation results indicated that the barrier at $1-2 \mathrm{MV} / \mathrm{m}$ is $\mathrm{MP}$ in zone 1 and the barrier at $12-19 \mathrm{MV} / \mathrm{m}$ is MP in zone 3 . Simulations done by Gonin and co-worker predicted a MP barrier at $0.7 \mathrm{MV} / \mathrm{m}$ in zone 1 and another barrier at $11-18 \mathrm{MV} / \mathrm{m}$ in zone 3 . Our simulation results predict two barriers, one at $0.6 \mathrm{MV} / \mathrm{m}$ in zone 1 and in the other at $10-30 \mathrm{MV} / \mathrm{m}$ in zone 3, respectively. There is a good agreement between MP barriers we predict and previous experiment and simulation results. The highest barrier we predict is above the experimentally achieved gradient. It should be mentioned that the original HOM coupler in the

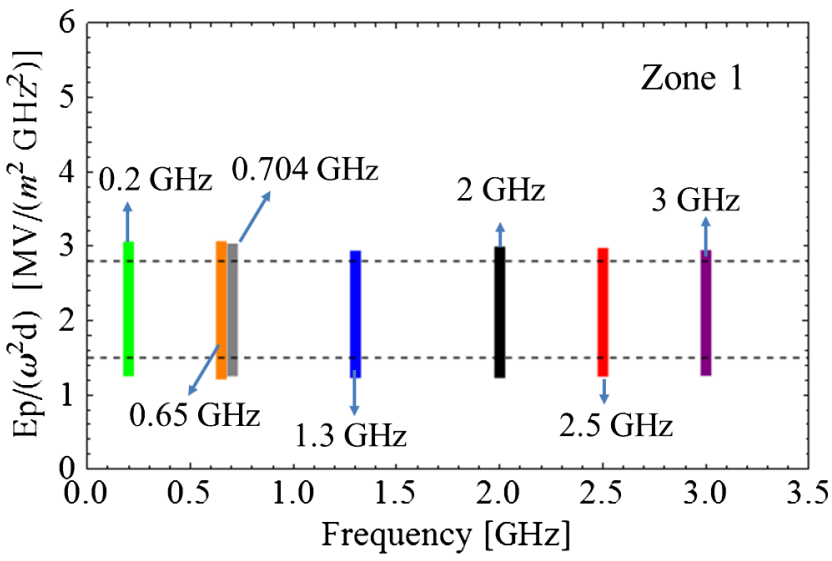

FIG. 11. Frequency scaling of zone $1 \mathrm{MP}$ in HOM couplers of scaled TESLA-type cavities. The dashed lines are the lower and upper bound from the analytical prediction based on the parallelplate model.

third harmonic cavity was ultimately redesigned and MP was successfully avoided [27].

\section{Zone 1 MP frequency scaling}

The electric field dominates in zone 1 of the ILC cavity. As can be seen in the following, the two-surface MP model is suitable for analyzing zone 1 over a broad frequency range. To study the frequency scaling, a series of cavities were scaled from a $1.3 \mathrm{GHz}$ TESLA-type onecell cavity. The notch filter is confirmed to work still for the scaled cavities. The frequency scaling of the zone 1 MP is shown in Fig. 11. The frequency scaling of the MP barrier is characterized by the range of $E_{p} / \omega^{2} d$, where $E_{p}$ is the local peak electric field, $\omega$ the angular rf frequency, and $d$ the gap dimension across the two local areas bombarded by MP electrons. As indicated earlier in Sec. V A, MP in zone 1 can be analytically treated as a two-surface MP based on the parallel-plate model, $\frac{m \omega^{2} d}{\sqrt{4+\pi^{2}} e}<E_{p}<\frac{m \omega^{2} d}{2 e}$. The lower and upper bound of the analytical prediction are shown as dashed lines in Fig. 11. It can be seen that the simulation results agreed fairly well

TABLE I. The local peak electric field $E_{p}$ and local gap $d$ of zone $1 \mathrm{MP}$ in scaled TESLA-type cavities. $E_{\text {acc }}$ is the corresponding acceleration gradient.

\begin{tabular}{lccl}
\hline \hline Freq $[\mathrm{GHz}]$ & $E_{\mathrm{acc}}[\mathrm{MV} / \mathrm{m}]$ & $E_{p}[\mathrm{MV} / \mathrm{m}]$ & $d[\mathrm{~mm}]$ \\
\hline 0.2 & $0.1-0.24$ & $0.019-0.043$ & 9.165 \\
0.65 & $0.3-0.7$ & $0.06-0.14$ & 2.82 \\
0.704 & $0.32-0.72$ & $0.067-0.15$ & 2.604 \\
1.3 & $0.6-1.6$ & $0.15-0.33$ & 1.41 \\
2 & $1.0-2.2$ & $0.19-0.42$ & 0.917 \\
2.5 & $1.2-2.8$ & $0.24-0.53$ & 0.733 \\
3 & $1.4-3.2$ & $0.29-0.62$ & 0.611 \\
\hline \hline
\end{tabular}




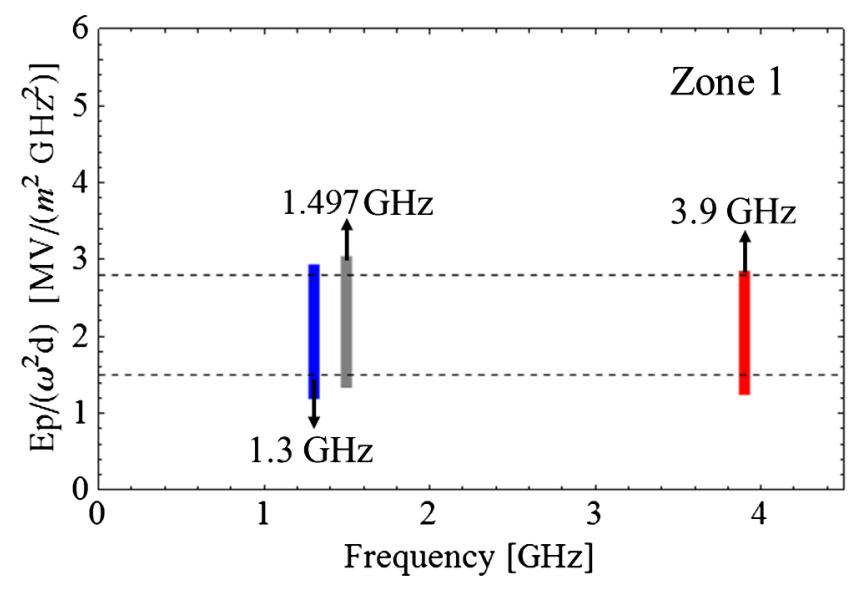

FIG. 12. Frequency scaling of zone $1 \mathrm{MP}$ in HOM couplers of real cavities including the $1.3 \mathrm{GHz}$ ILC cavity, $1.5 \mathrm{GHz}$ CEBAF upgrade cavity, and original $3.9 \mathrm{GHz}$ third harmonic cavity. The dashed lines are the lower and upper bound from the analytical prediction based on the parallel-plate model.

with the analytical result over a broad frequency range. A summary of the zone $1 \mathrm{MP}$ parameters of the scaled ILC cavities including the local electric field and the local gap separation is given in Table I.

Our new simulations of MP in real cavities confirm the existence of MP in zone 1 of the HOM coupler in the original $3.9 \mathrm{GHz}$ cavity. For the SNS high-beta $805 \mathrm{MHz}$ cavity, no zone $1 \mathrm{MP}$ is predicted because of the low impact energy in the range of 5-40 eV. Altogether, zone $1 \mathrm{MP}$ for real cavities including the $1.3 \mathrm{GHz}$ ILC cavity, $1.5 \mathrm{GHz}$ CEBAF upgrade cavity, and $3.9 \mathrm{GHz}$ cavity is summarized in Fig. 12. Also shown in Fig. 12 is the frequency scaling of the MP barrier as characterized by the range of $E_{p} / \omega^{2} d$. Again, the simulation results are found to agree fairly well with the analytical result over a broad frequency range. The zone $1 \mathrm{MP}$ parameters of the ILC, CEBAF, $3.9 \mathrm{GHz}$ cavities are given in Table II.

\section{Zone 3 MP frequency scaling}

Unlike zone 1, where a gap is formed by two flat surfaces, zone 3 is defined by more complicated surfaces. For the ILC, CEBAF upgrade, and SNS HOM coupler, the cross section of the HOM loop antenna has a racetrack

TABLE II. The zone 1 MP parameters of the ILC, CEBAF, $3.9 \mathrm{GHz}$ cavities.

\begin{tabular}{lccc}
\hline \hline Freq $[\mathrm{GHz}]$ & $E_{\mathrm{acc}}[\mathrm{MV} / \mathrm{m}]$ & $E_{p}[\mathrm{MV} / \mathrm{m}]$ & $d[\mathrm{~mm}]$ \\
\hline 1.3 & $0.6-1.6$ & $0.15-0.33$ & 1.41 \\
1.5 & $6-13$ & $0.228-0.472$ & 1.79 \\
3.9 & $0.55-0.65$ & $0.472-1$ & 0.6 \\
\hline \hline
\end{tabular}

(a)
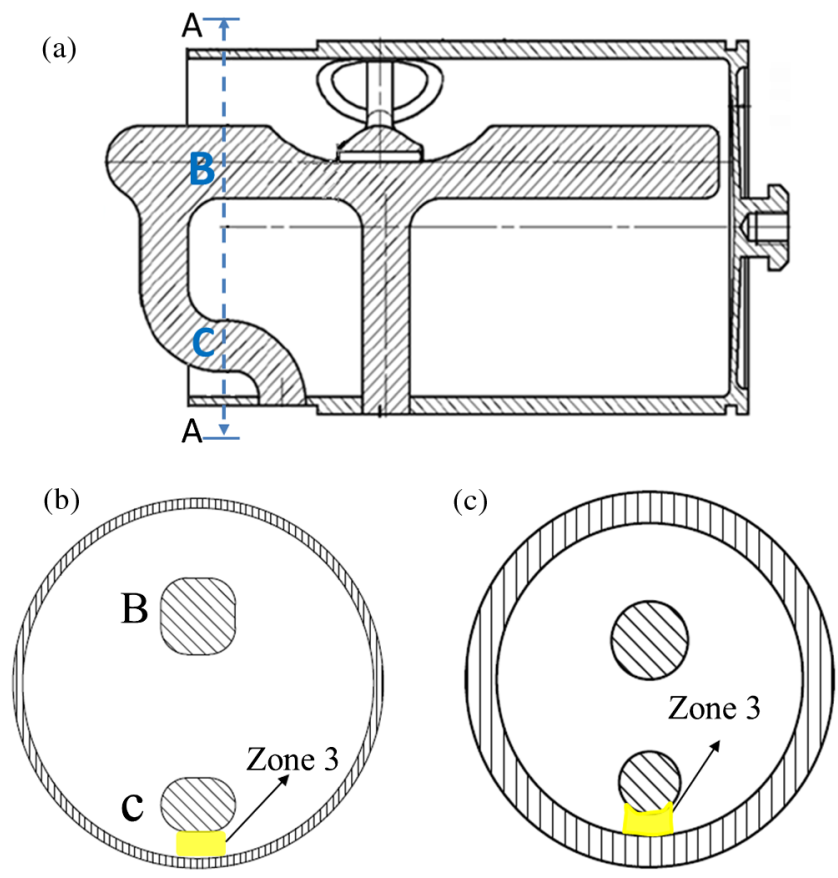

FIG. 13. (a) TESLA-style coaxial HOM coupler with sectioning line AA. (b) Cross section of ILC HOM coupler showing racetrack shaped HOM loop antenna. (c) Cross section of the 3.9 GHz original HOM coupler showing circular shaped HOM loop antenna.

shape. For the original $3.9 \mathrm{GHz} \mathrm{HOM}$ coupler, the cross section has a circular shape (see Fig. 13). The inner surface of the HOM can and the outer surface of the HOM loop antenna forms a two-surface region where MP is possible. From simulations, it is understood that the space occupied by resonant trajectories depends on the gradient. MP starts from region A at the MP onset gradient and moves toward region $\mathrm{B}$ with an increasing gradient (Fig. 14).

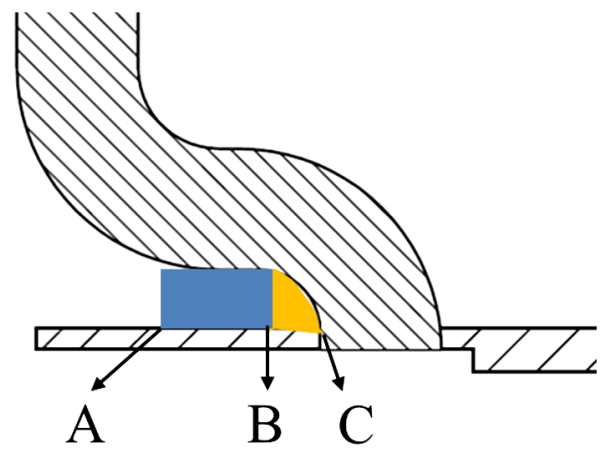

FIG. 14. Space occupied by two-surface MP in Zone 3. MP starts from region $\mathrm{A}$ at the onset gradient and moves toward region $B$ with an increasing gradient in ILC, CEBAF, and SNS cavity. For $3.9 \mathrm{GHz}$ original HOM coupler, the MP starts from A and moves toward $\mathrm{C}$. 


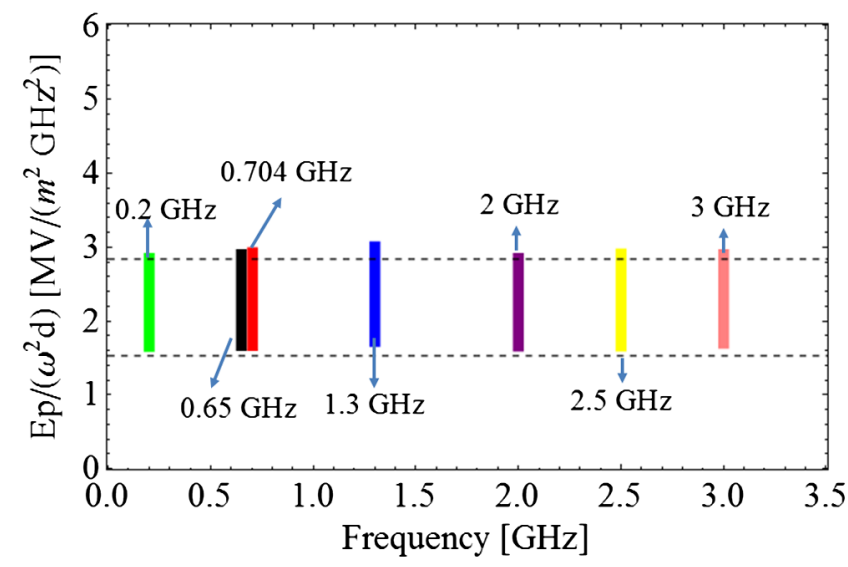

FIG. 15. Frequency scaling of zone 3 MP for scaled TESLAtype cavities.

The frequency scaling of the zone $3 \mathrm{MP}$ for scaled TESLA-shape cavities is shown in Fig. 15. Table III gives parameters of zone 3 first-order MP for the scaled TESLA-shape cavities. It should be noted that the scaled cavities are only for the purpose of analyzing the frequency scaling of zone 3 MP. HOM damping is not considered. It should be also noted that the broad gradient range covered by our simulation concerns only the MP dynamics for the completeness of the understanding. In reality, there are physical limits (such as the critical rf field of the superconductor) prohibiting a cavity from reaching a field beyond a threshold. From Fig. 15, we can see that the simulation results agreed fairly well with the two-surface MP analytical result over a broad frequency range.

Next we will show that the frequency scaling of zone 3 MP can be extended to other existing real cavities including SNS cavity ( $805 \mathrm{MHz}$ ), CEBAF upgrade cavity $(1.497 \mathrm{GHz})$, and $3.9 \mathrm{GHz}$ cavity. The results are shown in Fig. 16. It should be noted that, for the purpose of frequency scaling, the simulation of zone $3 \mathrm{MP}$ for the CEBAF upgrade cavity is extended well beyond the practical gradient range. The normalized local electric field $E_{p} /\left(\omega^{2} d\right)$ ranges from 1.5 to $3.1 \mathrm{MV} /\left(\mathrm{m}^{2} \mathrm{GHz}^{2}\right)$. It

TABLE III. The local peak electric field $E_{p}$ and local gap $d$ of zone $3 \mathrm{MP}$ in scaled TESLA-type cavities. $E_{\text {acc }}$ is the corresponding acceleration gradient.

\begin{tabular}{lccc}
\hline \hline Freq $[\mathrm{GHz}]$ & $E_{\mathrm{acc}}[\mathrm{MV} / \mathrm{m}]$ & $E_{p}[\mathrm{MV} / \mathrm{m}]$ & $d[\mathrm{~mm}]$ \\
\hline 0.2 & $7-22$ & $0.051-0.088$ & 19.5 \\
0.65 & $23-70$ & $0.167-0.29$ & 6 \\
0.704 & $26-75$ & $0.181-0.317$ & 5.54 \\
1.3 & $40-140$ & $0.345-0.602$ & 3 \\
2 & $70-218$ & $0.511-0.877$ & 1.95 \\
2.5 & $84-300$ & $0.639-1.12$ & 1.56 \\
3 & $105-340$ & $0.785-1.34$ & 1.3 \\
\hline \hline
\end{tabular}

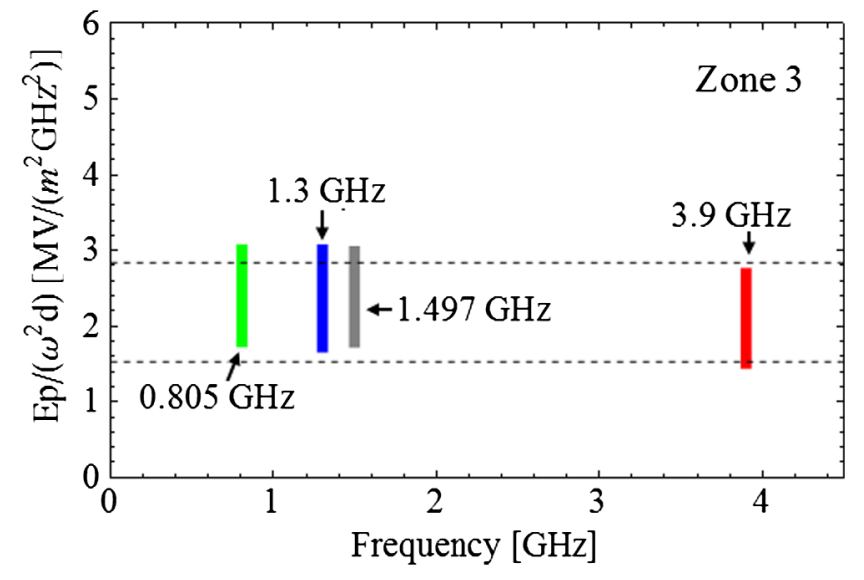

FIG. 16. Frequency scaling of zone $3 \mathrm{MP}$ in HOM couplers of real cavities. The dashed lines are the lower and upper bound for analytical prediction for two-plane first-order MP based on the parallel-plate model.

should be pointed out that the lower region [above $100 \mathrm{eV}$ impact energy, see Figs. 7(a) and 7(c)] MP is only possible in the space between line A and B (Fig. 13) where the gap separation is fixed in case of ILC, CEBAF upgrade, and SNS cavities; whereas in the case of the $3.9 \mathrm{GHz}$ cavity, the MP develops not only in the above region but also in regions beyond line $\mathrm{B}$ where the gap separation varies as the gradient is further raised. A summary of the zone 3 MP parameters including the local electric field and the local gap separation is given in Table IV.

From simulation results, "corner MP" is found in zone 2 from $133 \mathrm{MV} / \mathrm{m}$ to $155 \mathrm{MV} / \mathrm{m}$ (for theoretical MP study) in CEBAF upgrade cavity. No MP is found in zone 2 for $3.9 \mathrm{GHz}$ cavity. For ILC cavity, the MP is "corner MP" or one-point MP. No scaling law is found for MP in zone 2 .

\section{E. Discussion of MP modeling}

The initial energy of the emitted electrons can be varied changed (the default value is $2 \mathrm{eV}$ ). In our simulations, 3rd-order MP is found in Zone 3 in the ILC cavity, CEBAF upgrade cavity and SNS cavity with impact energy of

TABLE IV. Summary of the local peak electric field $E_{p}$ and local gap $d$ of zone $3 \mathrm{MP}$ in HOM couplers of different real cavities. $E_{\mathrm{acc}}$ is the corresponding acceleration gradient.

\begin{tabular}{lccc}
\hline \hline & $E_{\text {acc }}[\mathrm{MV} / \mathrm{m}]$ & $E_{P}[\mathrm{MV} / \mathrm{m}]$ & $d[\mathrm{~mm}]$ \\
\hline ILC & $40-140$ & $0.34-0.602$ & 3 \\
SNS high-beta & $25-35$ & $0.27-0.44$ & 5.83 \\
3.9 GHz & $10-30$ & $2.3-0.43$ & $1.4-0.48$ \\
CEBAF-Upgrade & $43-113$ & $0.47-0.79$ & 3.13 \\
\hline \hline
\end{tabular}


around $50 \mathrm{eV}$. The impact energy increases to $80 \mathrm{eV}$ when the initial energy is set to $5 \mathrm{eV}$.

Because of the fact that the initial energy of MP electrons is not very high, the magnetic force is much less compared to the electric force $(|q \vec{\nu} \times \vec{B}| \ll|q \vec{E}|)$. Therefore any initial angle deviation from the normal direction immediately disappears. In our simulation, the electric field is around several hundred $\mathrm{KV} / \mathrm{m}$ in zone 1 , zone 2 , and zone 3 , and the magnetic field magnitude is 8 orders less than the electric field magnitude. So, the electric field will play a dominant role during the initial emission stage. Because of this, it is expected that our simulation results do reflect the true physical process despite our limited selection of perpendicular emission. However, in the case of MP in a high magnetic field region, the initial angle effect is important and must be considered.

The secondary emission yield can be enhanced due to the glancing incident. This effect should be included in the analysis if measured SEY curves with glancing incident angles are available. The impact angle effect is not included in $\mathrm{ACE} 3 \mathrm{P}$ at the moment.

\section{CONCLUSION}

Simulations of MP in HOM couplers of the ILC and CEBAF $12 \mathrm{GeV}$ upgrade cavities have been carried out with the 3D code of Omega 3P and Track 3P developed by SLAC. For the ILC cavity, resonant MP electron trajectories exist from 0.6 to $1.6 \mathrm{MV} / \mathrm{m}$ in zone 1,21 to $34 \mathrm{MV} / \mathrm{m}$ in zone 2 , and 32 to $35 \mathrm{MV} / \mathrm{m}$ and above $40 \mathrm{MV} / \mathrm{m}$ in zone 3 . It is predicted that zone $3 \mathrm{MP}$ in an ILC cavity may limit the achievable gradient. Therefore, new HOM coupler designs are needed in further raising the gradient for the $1 \mathrm{TeV}$ upgrade of the ILC. For the CEBAF $12 \mathrm{GeV}$ upgrade cavity, resonant MP electron trajectories exist from 6 to $13 \mathrm{MV} / \mathrm{m}$ in zone 1 . This predicted MP barrier was not observed during the vertical cavity testing. It would be interesting to further assess this predicted barrier in the case of cavity operation with a beam. The frequency scaling of zone 1 and zone 3 MP over a broad frequency range is found well correlated with the two-surface MP predicted by the simple parallel plate model. The scaling law provides the possibility of assessing MP behaviors in a TESLA-style HOM coupler by only examining the local electromagnetic fields. Such a possibility allows rapid iterations in future HOM coupler design efforts for SRF cavities.

\section{ACKNOWLEDGMENTS}

We would like to thank Lixing Ge and Zenghai Li of SLAC for numerous discussions about the MP simulations using the Track 3P code. We thank Sang-Ho Kim of ORNL for discussions about the HOM coupler of SNS high beta cavity. Many thanks go to Ivan Gonin and Nikolay Solyak of FNAL for providing information about the $3.9 \mathrm{GHz}$ cavity HOM coupler model. We thank Haipeng Wang of
JLab for many useful discussions concerning this work. This work is authored by Jefferson Science Associates, LLC under U.S. DOE Contract No. DE-AC0506OR23177.

[1] P. T. Farnsworth, J. Franklin Inst. 218, 411 (1934).

[2] A. J. Hatch and H. B. Williams, J. Appl. Phys. 25, 417 (1954).

[3] C. M. Lyneis, H. A. Schwettman, and J. P. Turneaure, Appl. Phys. Lett. 31, 541 (1977).

[4] R. L. Geng, in Proceedings of the 20th Particle Accelerator Conference, Portland, OR, 2003 (IEEE, New York, 2003), p. 264-268.

[5] Practically, a hard MP barrier refers to a barrier that cannot be overcome by using the processing technique. Physically, a hard MP barrier is characterized by resonant trajectories with high phase stability and an impact energy in the range of 100-1000 eV. These conditions ensure a SEY larger than unity even for a clean surface and exponential growth in electron density that ultimately leads to rf breakdown.

[6] U. Klein and D. Proch, in Proceedings of the Conference on Future Possibilities for Electron Accelerators (University of Virginia, Charlottesville, VA, 1979), N1-17 (unpublished).

[7] P. Kneisel, R. Vincon, and J. Halbritter, Nucl. Instrum. Methods Phys. Res. 188, 669, (1981).

[8] J. Sekutowicz, in Proceedings of the 1993 Workshop on SRF, Newport News, VA, pp. 426-439 [http://accelconf .web.cern.ch/accelconf/SRF93/papers/srf93g04.pdf].

[9] R. L. Geng et al., in Proceedings of the 2008 Linear Accelerator Conference, LINAC08 OC, Victoria, British Columbia, pp. 879-881 (unpublished).

[10] G. Kreps et al., in Proceedings of the 2009 International Conference on RF Superconductivity, Berlin, Germany, pp. 289-291 [http://accelconf.web.cern.ch/AccelConf/ SRF2009/papers/tuppo036.pdf].

[11] A. Yamamoto, in Proceedings of the 2012 Linear Accelerator Conference, LINAC12 OC, Tel-Aviv, Israel, p. 787-791 (unpublished).

[12] I. Gonin et al., in Proceedings of the 22nd Particle Accelerator Conference, Albuquerque, NM, USA (IEEE, New York, 2007), WEPMN093, pp. 2248-2250.

[13] L. S. Cardman and L. Harwood, in Proceedings of the 22nd Particle Accelerator Conference, Albuquerque, NM (IEEE, New York, 2007), MOZBKIO, pp. 58-62.

[14] C. E. Reece et al., in Proceedings of the 13th International Conference on RF Superconductivity, Beijing, China, 2007, pp. 536-539 [http://accelconf.web.cern.ch/ AccelConf/srf2007/PAPERS/WEP31.pdf].

[15] F. Marhauser, in Proceedings of the International Workshop on HOM Damping in Superconducting RF Cavities, Cornell University, 2010 [http://www.Ins.cornell.edu/ Events/HOM10/Agenda.html].

[16] F. Marhauser et al., in Proceedings of the 2nd International Particle Accelerator Conference, San Sebastián, Spain (EPS-AG, Spain, 2011), MOPC112, pp. 337-339.

[17] Sang-ho Kim et al., Proceedings of the 2006 Linear Accelerator Conference, LINACO6 OC, Knoxville, TN, 
pp. 770-772 [http://accelconf.web.cern.ch/AccelConfl06/ PAPERS/THP081.PDF].

[18] K. Ko et al., in Proceedings of the 25th International Linear Accelerator Conference, LINAC-2010, Tsukuba, Japan (KEK, Tsukuba, Japan, 2011), FR101, pp. 1028-1032.

[19] L. Ge et al., in Proceeding of the 3rd International Particle Accelerator Conference, New Orleans, LA, IPAC-2012 (IEEE, Piscataway, NJ, 2006), WEPPC068, pp. 2366-2368.

[20] C. Ng et al., in Proceeding of the 10th European Particle Accelerator Conference, Edinburgh, Scotland, EPAC2006 (EPS-AG, Edinburgh, Scotland, 2006), THXFI01, pp. 2763-2767.

[21] Z. Li (private communication).

[22] H. Padamsee, J. Knobloch, and T. Hays, RF Superconductivity for Accelerators (John Wiley \& Sons, New York, 1998), p. 184.
[23] G. Wu et al., in Proceedings of the 12th International Workshop on RF Superconductivity, Ithaca, New York, 2005, THP58, pp. 600-603 [http://accelconf.web.cern.ch/ AccelConf/SRF2005/papers/thp58.pdf].

[24] Thomas P. Wangler, RF Linear Accelerators (Wiley-VCH, New York, 2008), pp. 159-161.

[25] D. Kostin et al., in Proceedings of the 15th International Workshop on RF Superconductivity, Chicago, IL, 2011, THPO003, pp. 691-694 [http://accelconf.web.cern.ch/ AccelConf/SRF2011/papers/thpo003.pdf].

[26] D. Reschke et al., in Proceedings of the 15th International Workshop on RF Superconductivity, Chicago, IL, THPO046, pp. 490-494 [http://accelconf.web.cern.ch/ AccelConf/SRF2011/papers/tupo046.pdf].

[27] T. Khabiboulline et al., in Proceedings of the 22nd Particle Accelerator Conference, PAC-2007, Albuquerque, NM (IEEE, New York, 2007),WEPMN098, pp. 2259-2261. 